\title{
Highly Anisotropic Transport in the Integer Quantum Hall Effect
}

\author{
W. Pan ${ }^{1,2}$, H.L. Stormer ${ }^{3,4}$, D.C. Tsui ${ }^{1}$, L.N. Pfeiffer ${ }^{4}$, K.W. Baldwin ${ }^{4}$, and K.W. West ${ }^{4}$ \\ ${ }^{1}$ Department of Electrical Engineering, Princeton University, Princeton, New Jersey 08544 \\ ${ }^{2}$ National High Magnetic Field Laboratory, Tallahassee, Florida 32310 \\ ${ }^{3}$ Department of Physics and Department of Applied Physics, Columbia University, New York, New York 10027 \\ ${ }^{4}$ Bell Labs, Lucent Technologies, Murray Hill, New Jersey 07974
}

(October 31, 2018)

\begin{abstract}
At very large tilt of the magnetic $(B)$ field with respect to the plane of a two-dimensional electron system the transport in the integer quantum Hall regime at $\nu=4,6$, and 8 becomes strongly anisotropic. At these filling factors the usual deep minima in the magneto-resistance occur for the current flowing perpendicular to the in-plane $B$ field direction but develop into strong maxima for the current flowing parallel to the in-plane $B$ field. The origin of this anisotropy is unknown but resembles the recently observed anisotropy at half-filled Landau levels.
\end{abstract}

Strongly correlated electronic systems often exhibit stripe phases [1]. In two-dimensional electron systems (2DES) such a stripe phase is believed to be at the origin of the recently observed electronic transport anisotropy at half-fillings of high Landau levels [2] 8]. At Landau level filling factors $\nu=9 / 2,11 / 2,13 / 2$, etc. the magneto-resistance is a maximum along one current direction, whereas it is a minimum when the current direction is rotated by $90^{\circ}$ within the plane of the sample. In a purely perpendicular magnetic field $(B)$ the direction of anisotropy is pinned to the crystal lattice [3. 4 , but re-orients itself when an in-plane $B$ field $\left(B_{i p}\right)$ is added by tilting the sample. At large $B_{i p}$ the easy-axis of anisotropy in the plane of the sample (the direction of minimum resistance) is always perpendicular to $B_{i p}$ [5.6. Although the nature of this anisotropy remains uncertain, experimental data [2, 8] and theoretical models [9 21] point to the formation of a unidirectional charge density wave, often referred to as the "stripe phase", or to a state akin to a liquid crystal phase [11]. A very similar anisotropy is also observed at $\nu=5 / 2$ and $\nu=7 / 2$ in the second Landau level under large $B_{i p}$ [5]6]. Modeling [22] suggests that an electronic anisotropic phase, not unlike the one at half-fillings of higher Landau levels, has been induced by the in-plane $B$ field.

So far, anisotropy has only been observed at half-filled Landau levels. In this letter, we present data that show strong electronic transport anisotropies at fully filled Landau levels. They are created by the very strong inplane $B$ fields at very large tilt in the regime of the integral quantum Hall effect (IQHE) at $\nu=4,6$, and 8 . The origin of these anisotropies is unknown, although, phenomenologically, they resemble the anisotropies at halffilled Landau levels: the magneto-resistance is a minimum when the current is perpendicular to $B_{i p}$ and a maximum when the current is along $B_{i p}$. A striped spin density wave phase may be at the origin of these new observations.

Our sample consists of a $350 \AA$ wide GaAs quantum well embedded into $\mathrm{Al}_{.24} \mathrm{Ga}_{.76} \mathrm{As}$ and delta-doped from both sides at a distance of $490 \AA$. The specimen has a size of $5 \mathrm{~mm} \times 5 \mathrm{~mm}$ and is contacted via eight indium contacts placed symmetrically around the perimeter. The electron density is established after illuminating the sample with a red light-emitting diode at $\sim 4.2 \mathrm{~K}$ and, within limits, the density can be tuned by exposure time. At an electron density of $n=4.2 \times 10^{11} \mathrm{~cm}^{-2}$ two electrical subbands are populated having densities $n_{0} \sim 3.1 \times 10^{11}$ $\mathrm{cm}^{-2}$ and $n_{1} \sim 1.1 \times 10^{11} \mathrm{~cm}^{-2}$ as determined by Fourier analysis of the low-field Shubnikov-de Haas oscillations. All angular dependent measurements are carried out in a dilution refrigerator equipped with an in-situ rotator placed inside a 33 Tesla resistive magnet. We define the axis of rotation as the $y$-axis. Consequently, the in-plane field, $B_{i p}$, extends along the $x$-axis when the sample is rotated.

We have measured $R_{x x}$ and $R_{y y}$, which differ only in the in-plane current direction, at more than 10 tilt angles $(\theta)$ between $0^{\circ}$ and $90^{\circ} . R_{x x}$ represents the direction for which, under tilt, the current runs along $B_{i p}$. Figure 1 shows data at five selected angles, from $\theta=81.1^{\circ}$ to $84.4^{\circ}$. At $\theta=0^{\circ}$ (not shown) both $R_{x x}$ and $R_{y y}$ vanish at $\nu=6$ as expected for an isotropic quantum Hall state. As $\theta$ is increased towards $81.1^{\circ}$, both $R_{x x}$ and $R_{y y}$ remain vanishingly small at $\nu=6$, although the widths of the resistance minima and of the Hall plateau shrink with increasing $\theta$. Very generally, such an angular dependence is readily understood for the spin-unpolarized $\nu=6$ state. While the $\nu=6$ state always occurs at the same perpendicular magnetic field, $B_{\text {perp }}$, the total magnetic field at tilt angle, $\theta$, increases as $B_{\text {tot }}=B_{\text {perp }} / \cos (\theta)$. Since the electron spin experiences $B_{t o t}$, the Zeeman splitting of all Landau levels increases with increasing $\theta$. This leads to a reduction of the energy gap at $\nu=6$ and a shrink- 
ing width and depth (not visible on the linear scale of Fig. 1) of the $R_{x x}$ and $R_{y y}$ minima. Eventually, this leads to a collapse and disappearance of the $\nu=6 \mathrm{IQHE}$ state. Indeed, at $\theta=83.3^{\circ}, R_{x x}$ has turned from a deep minimum into a strong peak and the usual Hall plateau has vanished. Therefore, the disappearance of $R_{x x}$ can be rationalized as the closing of the $\nu=6$ energy gap. However, very surprisingly, the electrical transport turns out to be strongly anisotropic. In contrast to $R_{x x}$, which shows a strong maximum at this angle and filling factor, $R_{y y}$, continues to shows a strong minimum at $\nu=$ 6. Just as in the case of half-fillings [5, 6] the easy-axis of this anisotropy at full-filling factors is perpendicular to $B_{i p}$. The direction of anisotropy is not dependent on the orientation of the crystallographic axis with respect to the in-plane field, as we determined by performing the same experiments on the same specimen mounted in a configuration rotated by $90^{\circ}$ about the sample normal. Furthermore, none of the resistance measurements showed any hysteresis as a function of the sweep direction of the $B$ field. Finally, in the anisotropic regime, the generally strong Hall plateau at $\nu=6$ disappears for both directions of current.

This is the first time that such an anisotropy has been observed in a state as robust as an IQHE state. To learn more about this anisotropic state we perform $T$ dependent studies of $R_{x x}$ and $R_{y y}$. For comparisons, we choose $\theta=81.1^{\circ}$, where the electronic transport is isotropic, and $\theta=83.3^{\circ}$, where transport is strongly anisotropic. in Figure $2 \mathrm{a}$ and Figure $2 \mathrm{~b}$ we show three representative traces of $R_{x x}$ and $R_{y y}$. At $\theta=81.1^{\circ}, R_{x x}$ and $R_{y y}$ exhibit the usual activated behavior: the value of both resistances increases with increasing $T$. On the other hand, at $\theta=83.3^{\circ}, R_{x x}$ and $R_{y y}$ behave oppositely: $R_{x x}$ decreases whereas $R_{y y}$ increases with increasing $T$. The $T$-dependencies are quantified in Figure 2c and Figure $2 \mathrm{~d}$, where $R_{x x}$ and $R_{y y}$ are shown on Arrhenius plots. At $\theta=81.1^{\circ}, R_{x x}$ and $R_{y y}$ show well-behaved activated behavior yielding a single energy gap of $\Delta \sim 1 \mathrm{~K}$ for both current directions [23]. On the other hand, the data for $R_{x x}$ and $R_{y y}$ at $\theta=83.3^{\circ}$ show no longer activated behavior. $R_{x x}$ and $R_{y y}$ appear to start from similar values at high temperature but then diverge from each other roughly exponentially with exponents of similar magnitude but opposite sign. At the lowest temperatures both resistances assume an approximately $T$-independent behavior. This dependence is qualitatively the same as the $T$-dependence of the anisotropic state at $\nu=9 / 2$ [, [17].

The remarkable anisotropy found in the IQHE is not limited to the $\nu=6$ state. Similar anisotropies are observed at filling factors $\nu=4$ and $\nu=8$. Figure 3 shows the $\nu=8$ and $\nu=4$ anisotropy in the same sample at slightly different densities, tuned by applying different doses of light. We have not performed a systematic study of these states.

The cause of the anisotropy at integral quantum Hall states is unknown. Before speculating about the origin of this new phenomenon it is instructive to consider in more detail the single particle states in this two-electric subband specimen. Figure 4a shows the usual Landau fan diagram for a density of $4.2 \times 10^{11} \mathrm{~cm}^{-2}$. The Zeeman splitting is enhanced by a factor of 10 to be visible. The position of the Fermi level, $E_{f}$, is indicated by a heavy line. Clearly, in the vicinity of $\nu=4,6$ and 8, Landau levels from both electric subbands contribute and $E_{f}$ jumps between levels of different origin. Using such a simple single-particle picture and a 2DES of zero thickness with densities appropriate for the data of Figs. 1 and 3, one would expect the gaps at $\nu=4,6$ and 8 to close at $\theta=$ $83.4^{\circ}, 88.4^{\circ}$ and $88.3^{\circ}$, respectively. These values differ from experiment, especially in the case of the $\nu=6$ and $\nu=8$ states.

The discrepancy is largely the result of the neglect of exchange and of the thickness of the wave function. In the remainder we focus on the state at $\nu=6$, which we studied most extensively and which shows the strongest anisotropy in experiment. We expect similar arguments to hold for $\nu=4$ and $\nu=8$. Figure $4 \mathrm{~b}$ shows the result of a self-consistent local-density-approximation calculation [24] performed for a density $n=4.2 \times 10^{11} \mathrm{~cm}^{-2}$ at a filling factor $\nu=6$ as a function of $B_{i p}$. The gap at $\nu=$ 6 (shaded region) undergoes strong variations, comes almost to a close at $B_{i p} \sim 2.5 \mathrm{~T}$ (not shown), and vanishes at $B_{i p} \sim 18.5 \mathrm{~T}$ due to level crossing. The experimental value of $B_{i p}$ for the strong anisotropy is $\sim 25 \mathrm{~T}$. However, we consider the theoretical result of $\sim 18.5 \mathrm{~T}$ to be sufficiently close to $\sim 25 \mathrm{~T}$ to attribute the disappearance of the energy gap at $\nu=6$ in Fig. 1 to the crossing of spinsplit Landau levels originating from different electrical subbands $(i=1,2)$. This provides a rational for the appearance of novel features in the data at this filling factor and angle. However, none of such level crossing considerations can explain the observed anisotropy, which represents the remarkable finding in our data. The origin of this phenomenon must be the result of correlated electron behavior.

Previously, large electrical anisotropies have only been observed at half-filled Landau levels [2 8]. It is believed that there the electron system spontaneously breaks into striped domains of alternating filling factors such as $\nu=4$ and $\nu=5$ around $\nu=9 / 2$ [21. Given the similarity of the observed properties of the anisotropic phases around $\nu=9 / 2$ and $\nu=6$ one might speculate on a similar underlying striped geometry. The driving force behind the phase separation in the $\nu=9 / 2$ case is exchange. The energetic gain from breaking into domains of $\nu=4$ and $\nu=5$ is counteracted by a strong electrostatic cost for creating an inhomogeneous charge distribution. This is the reason for the formation of very narrow stripes of $\nu=4$ and $\nu=5$ states, which are only a few magnetic lengths wide. A phase, consisting of stripes around $\nu=$ 6 , would carry a much smaller, electrostatic burden. 
At the point of collapse of the $\nu=6$ energy gap in Fig. 4b two electronic configurations are degenerate. At $B_{i p}$ smaller than the level crossing in Fig. $4 \mathrm{~b}$ the electrons occupy three spin-unpolarized levels emanating from the lowest three Landau levels $(N=0,1$, and 2$)$ of the lower electronic subband, $i=1$ 25]. (Note, an earlier anticrossing at $B_{i p} \sim 2.5 \mathrm{~T}$ exchanges states $i=1, N=2$ and $i=2, N=0)$. The total system is spin-unpolarized (3 spin-up, 3 spin-down). At $B_{i p}$ larger than the level crossing in Fig. 4b the electrons occupy only two spinunpolarized levels emanating from the lowest two Landau levels $(N=0,1)$ of the lower electronic subband $(i=1)$. In addition, they occupy the spin-up states (solid lines) of two levels emanating from the $i=1, N=2$ and the $i=$ $2, N=0$ states. There, the total system is partially spinpolarized (4 spin-up, 2 spin-down). In the vicinity of the level crossing in Fig. 4b, a phase separation of the electronic system [26, 27] into spin-unpolarized and partially spin-polarized domains may occur driven by exchange. A very small gain in exchange energy may suffice, since the charge density in both configurations is identical and, to first order, there is no associated electrostatic cost.

Such a pattern resembles the pattern of a spin-density wave, SDW. The existence of an in-plane magnetic field and the so-induced coupling of spin and orbital motion will energetically favor a given orientation of the stripes with respect to $B_{i p}$. The resulting stripe phase of alternating IQHE configurations is bound to have one-dimensional edge-states along its interface between neighboring domains, which carry the electric current in a highly anisotropic fashion. This transport pattern would be analogous to the pattern invoked in the stripe phases that are believed to form at half-fillings of Landau levels, such as $\nu=9 / 2$ and $13 / 2$ and believed to be responsible for the anisotropic electronic behavior. However, without the application of other experimental techniques and without a detailed theoretical investigation this picture remains speculative.

In summary, we have observed strongly anisotropic transport under high in-plane magnetic field in the regime of the IQHE in a quantum well sample with two occupied electrical subbands. Phenomenologically, the data have much in common with the previously discovered anisotropy at half-fillings of high Landau levels. From a simple level crossing picture we conjecture that a novel striped spin-density wave may be at the origin of this phenomenon.

We would like to thank E. Palm and T. Murphy for experimental assistance, E. P. De Poortere, S. P. Shukla, and E. Tutuc for the help in numerical calculation, and N. Bonesteel, R. R. Du, A.H. MacDonald, N. Read, and $\mathrm{K}$. Yang for useful discussion. We are indebted to $\mathrm{T}$. Jungwirth for providing the energy level scheme of Figure 4b. A portion of this work was performed at the National High Magnetic Field Laboratory, which is supported by NSF Cooperative Agreement No. DMR-9527035 and by the State of Florida. D.C.T. and W.P. are supported by the DOE and the NSF.

[1] V.J. Emery, E. Fradkin, S.A. Kivelson, and T.C. Lubensky, Phys. Rev. Lett. 85, 2160 (2000).

[2] H.L. Stormer, R.R. Du, D.C. Tsui, L.N. Pfeiffer, and K.W. West, Bull. Amer. Phys. Soc. 38, 235 (1993).

[3] M. P. Lilly, K.B. Cooper, J.P. Eisenstein, L.N. Pfeiffer, and K.W. West, Phys. Rev. Lett. 82, 394 (1999).

[4] R.R. Du, D.C. Tsui, H.L. Stormer, L.N. Pfeiffer, K.W. Baldwin, and K.W. West, Solid State Commun. 109, 389 (1999).

[5] W. Pan, R.R. Du, H.L. Stormer, D.C. Tsui, L.N. Pfeiffer, K.W. Baldwin, and K.W. West, Phys. Rev. Lett. 83, 820 (1999).

[6] M.P. Lilly, K.B. Cooper, J.P. Eisenstein, L.N. Pfeiffer, and K.W. West, Phys. Rev. Lett. 83, 824 (1999).

[7] M. Shayegan, H.C. Manoharan, S.J. Papadakis, and E.P. De Poortere, Physica E, 6, 40 (2000).

[8] W. Pan, T. Jungwirth, H.L. Stormer, D.C. Tsui, A.H. MacDonald, S.M. Girvin, L. Smrčka, L.N. Pfeiffer, K.W. Baldwin, and K.W. West, Phys. Rev. Lett. 85, 3257 (2000).

[9] A. A. Koulakov, M. M. Fogler, and B. I. Shklovskii, Phys. Rev. Lett. 76, 499 (1996); M.M. Fogler, A.A. Koulakov, and B.I. Shklovskii, Phys. Rev. B 54, 1853 (1996); M.M. Fogler and A.A. Koulakov, Phys. Rev. B 55, 9326 (1997).

[10] R. Moessner and J. T. Chalker, Phys. Rev. B 54, 5006 (1996).

[11] E. Fradkin and S. A. Kivelson, Phys. Rev. B 59, 8065 (1999).

[12] H.A. Fertig, Phys. Rev. Lett. 82, 3593 (1999).

[13] E.H. Rezayi, F.D.M. Haldane, and Kun Yang, Phys. Rev. Lett. 83, 1219 (1999).

[14] S.H. Simon, Phys. Rev. Lett. 83, 4223 (1999).

[15] T. Jungwirth, A.H. MacDonald, L. Smrčka, and S.M. Girvin, Phys. Rev. B 60, 15574 (1999).

[16] T. Stanescu, I. Martin, and P. Phillips, Phys. Rev. Lett. 84, 1288 (2000).

[17] E. Fradkin, S.A. Kivelson, E.Manousakis, and K. Nho, Phys. Rev. Lett. 84, 1982 (2000).

[18] N. Maeda, Phys. Rev. B 61, 4766 (2000).

[19] A.H. MacDonald and M.P.A. Fisher, Phys. Rev. B 61, 5724 (2000).

[20] F. von Oppen, B.I. Halperin, and A. Stern, Phys. Rev. Lett. 84, 2937 (2000).

[21] R. Côté and H.A. Fertig, Phys. Rev. B 62, 1993 (2000).

[22] E.H. Rezayi and F.D.M. Haldane, Phys. Rev. Lett. 84, 4685 (2000).

[23] $R_{x x}$ is about three times larger than $R_{y y}$ at the same temperature. This level of initial anisotropy is not uncommon in 2DES, whether they are patterned or cleaved into squares. It is probably due to small density variations leading to current inhomogeneities. However, it cannot be the origin of the strong anisotropy since the 
strong anisotropy observed at $\nu=4,6$, and 8 is pegged to $B_{i p}$ and not to any particular sample direction.

[24] T. Jungwirth, unpublished.

[25] Strictly speaking, the separation into subband levels and Landau levels only holds at zero tilt angle. At non-zero tilt they are mixed and one can use this nomenclature only approximately.

[26] G.F. Giuliani and J.J. Quinn, Phys. Rev. B 31, 6228 (1985).

[27] Sudhakar Yalagadda, Phys. Rev. B 44, 13101 (1991).

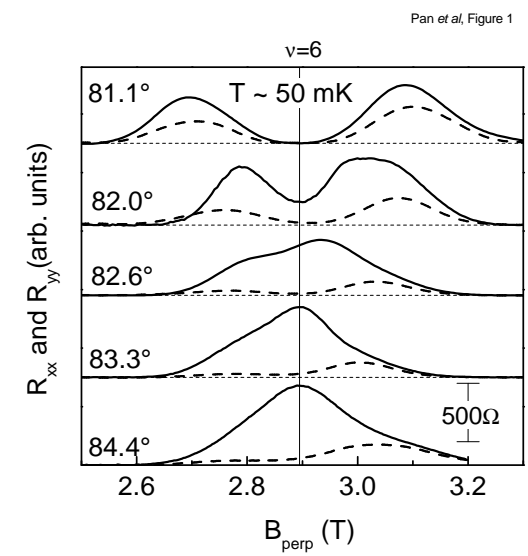

FIG. 1. $\quad R_{x x}$ (solid lines) and $R_{y y}$ (dashed lines) around $\nu$ $=6$ at $T \sim 50 \mathrm{mK}, I=10 \mathrm{nA}$ and for five tilt angles, from $\theta=81.1^{\circ}$ to $\theta=84.4^{\circ}$. For $R_{x x}$ the current runs along the in-plane magnetic field. $n=4.2 \times 10^{11} \mathrm{~cm}^{-2}$.

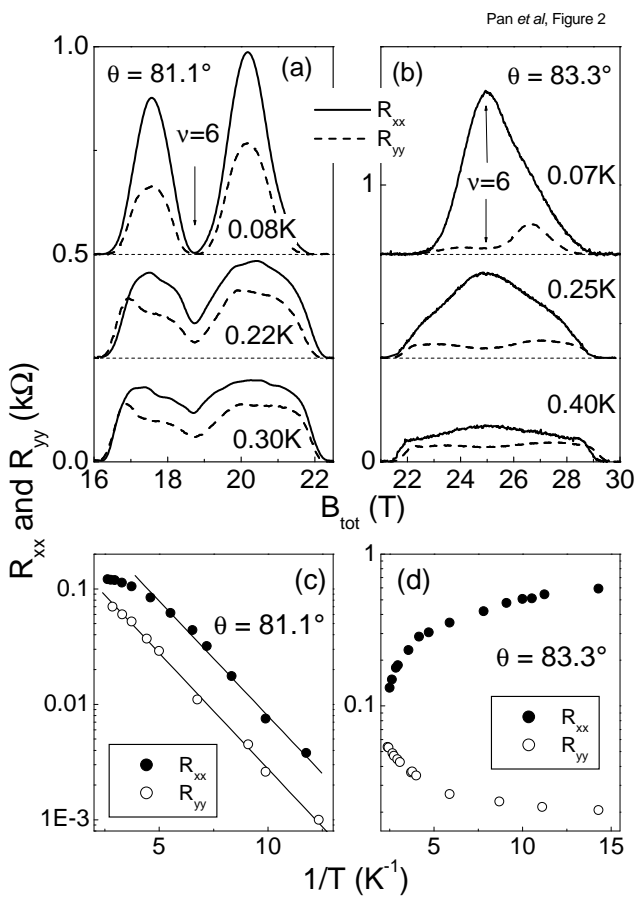

FIG. 2. Panels (a) and (b): Temperature dependence of $R_{x x}$ (solid lines) and $R_{y y}$ (dashed lines) at tilt angles $\theta$ $=81.1^{\circ}(\mathrm{a})$ and $83.3^{\circ}(\mathrm{b})$ and three different temperatures each. Traces are shifted vertically for clarity. The position of the $\nu=6$ filling factor is indicated. Panels (c) and (d): Corresponding Arrhenius plots for $R_{x x}$ and $R_{y y}$ at $\nu=6$ at the tilt angles of the panels above. The straight lines in panel (c) are a linear fit to the data [23]. The energy gap is $\sim 1 \mathrm{~K}$ for $R_{x x}$ and $R_{y y}$.

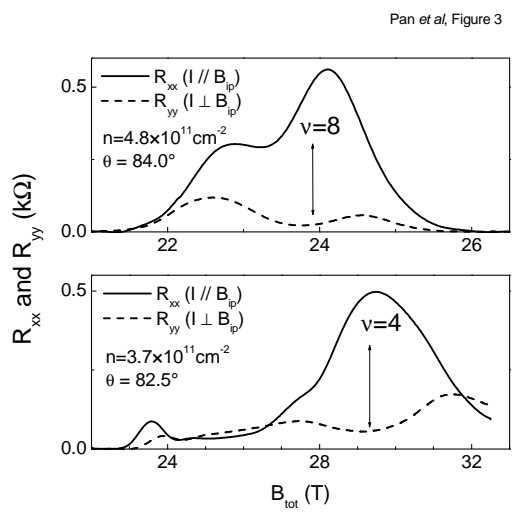

FIG. 3. (a) Anisotropic transport around $\nu=8$ at $\theta=$ $84.0^{\circ}$. (b) Anisotropic transport around $\nu=4$ at $\theta=82.5^{\circ}$. The sample densities are slightly different from Fig. 1 and have been adjusted by different light exposure. For $R_{x x}$ the current runs along the in-plane magnetic field.
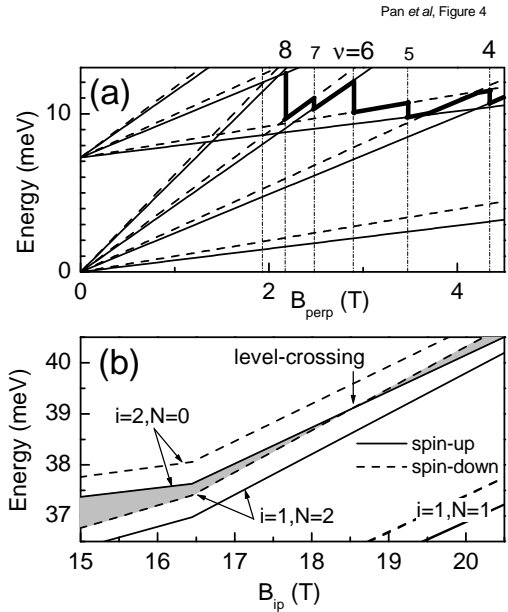

FIG. 4. Panel (a): Simple Landau fan diagram for the two-electric subband sample of density $n=4.2 \times 10^{11}$ $\mathrm{cm}^{-2}$. The Zeeman splitting is enhanced by a factor of 10 to be visible. The position of the Fermi level is indicated by heavy line. Panel (b): Result of self-consistent local-density-approximation calculation [24] at $\nu=6$ and as a function of in-plane magnetic field, $B_{i p}$, at the same density as panel (a). The electric subband index, $i$, and the Landau level index, $N$, are indicated. The shaded area represents the gap at $\nu=6$. 